\title{
Migration of Neglected Vaginal Pessaries: A Case Report
}

\author{
Hyun A Bae' ${ }^{1}$, Jeong In Choi', Soo-Ho Chung ${ }^{1}$, Jae Hong Sang ${ }^{1 * \mathbb{C}}$
}

\begin{abstract}
Introduction: Vaginal pessaries are commonly used to manage pelvic organ prolapse. Pessaries are usually tolerated although some severe complications can occur in cases of negligence, including vesicovaginal and rectovaginal fistulas.

Case Presentation: This is a report of two cases of a neglected vaginal pessary and migration into the rectovaginal space to form a vaginal orifice. Based on examinations, no vaginal pessaries in both cases migrated into the rectum. These pessaries were removed by cutting and rotating movements. Finally, the two patients were discharged after surgery without any complications.

Conclusions: Neglected pessaries can lead to serious complications. Thus, patient education, local estrogen treatment, and careful follow-ups are important to prevent such complications.

Keywords: Pessary, Migration, Uterine prolapse, Complications
\end{abstract}

\section{Introduction}

A pessary is used to reduce the protrusion of pelvic structures into the vagina. Pessaries are usually made from medical silicone. There are various types of pessaries, including rings, cubes, inflatoballs, donuts, and gel horns. Due to its minimal invasiveness, a vaginal pessary is suitable for patients who prefer non-surgical treatment of a uterovaginal prolapse or are unfit for surgery due to old age or comorbidities (1). A success rate of $62 \%$ has been reported in patients with stages III and IV prolapse (2). The most common side effect of a pessary is vaginal irritation (3), but pelvic or abdominal pain, vaginal discharge, odor, and bleeding can occur as well (4). Rare complications include urinary, rectal, and genital complications such as fistulas, fecal impaction, hydronephrosis, and urosepsis (5). Severe complications tend to occur in patients who neglect a pessary after placement. A pessary requires self-care by patients, including the periodic removal for cleaning and replacement. In addition, the routine office follow-up is mandatory because of the side effects and complications $(6,7)$. However, there is no consensus about the optimal frequency of care in the literature. Some physicians follow up for 2 weeks, then every 3 months for the first year, and every 6 months thereafter (8) whereas others suggest a follow-up of every 9-12 months as long as there are no adverse symptoms (1).

\section{Case 1}

A 79-year-old multiparous woman complained of vaginal spotting. She was diagnosed with hypertension and diabetes. She received an intra-vaginal sling and underwent cystocele repair due to a uterovaginal prolapse
11 years ago. One year after the surgery, the uterovaginal prolapse relapsed, and a ring pessary was inserted accordingly. The patient did not visit our clinic for 6 years.

A pessary was noted upon inspection with a speculum, but it did not move due to adhesions. It was located under the cervix, and the vagina was stuck together. Transvaginal ultrasonography revealed normal results. A magnetic resonance image was taken to visualize the retained pessary (Figure 1A). The patient was hospitalized, and blood testing was conducted for her. The white blood cell (WBC) and the C-reactive protein (CRP) contents were 9600 and 0.36, respectively. An estriol and antibiotic capsule was inserted transvaginally. Then, the neglected pessary was removed under general anesthesia. The route of pessary impaction was checked using hysteroscopy and it was grossly observed (Figure 1B) to assure that it was in the dorsal lithotomy position. The pessary was cut using an orthopedic bone cutter and a number 11 blade, rotated, and removed from the vagina. The vaginal tissue around the retained pessary was not damaged (Figures $1 \mathrm{C}$ and 1D), and a vaginal dressing was applied with betadine. The patient was discharged on the day of surgery.

She visited the clinic after 1 week and reported no vaginal bleeding or vaginal prolapse. Adhesions were noted in the vagina during a physical examination.

\section{Case 2}

An 80-year-old multiparous woman presented with lower abdominal pain. She had a history of hypertension, diabetes, and dementia. A pessary had been inserted 7 years prior to her presentation due to uterine prolapse. She visited our clinic 3 months after the pessary was placed

Received 17 November 2019, Accepted 22 April 2020, Available online 15 August 2020

'Department of Obstetrics and Gynecology, Soonchunhyang University College of Medicine, Bucheon, South Korea.

*Corresponding Author: Jae Hong Sang, MD, Ph.D., Tel: +82-32-621-5055, Fax:+82-2-6008-6874, Email: 77215@schmc.ac.kr 


\section{Key Messages}

- A pessary requires routine office follow-ups, otherwise, it could lead to severe complications such as neglected pessary.

because of the expulsion of the pessary. The pessary was sterilized and reinserted at that time. However, she did not visit the clinic thereafter. Upon physical examinations with a speculum and a colposcope, the pessary was observed to be stuck in the vaginal wall and was not moveable (Figure 2A). Blood testing was done, and the patient's WBC and CRP contents were 16740 and 16.22, respectively. She was hospitalized, and $1 \mathrm{~g}$ cefotetan was administered three times a day for 12 days. Sigmoidoscopy was performed to check if a recto-vaginal fistula was present, but none was found, and the CRP level decreased to 0.52 after antibiotic therapy.

The visible field was checked with a speculum under general anesthesia with the patient in the dorsal lithotomy position. The observed partial pessary was cut with a number 11 blade, rotated, and removed from the vagina (Figures $2 \mathrm{~B}$ and $2 \mathrm{C}$ ). A vaginal wall orifice was noted after removing the pessary (Figure 2D). A digital rectal exam was conducted, but no recto-vaginal fistula was observable. The patient was discharged the day after the surgery and treated with $100 \mathrm{mg}$ cefdinir three times a day for 7 days. She visited the clinic 1 week later and did not complain of any symptoms.

\section{Discussion}

A pessary is a good alternative for patients with an uterovaginal prolapse who are unfit for a surgical treatment. However, these patients are usually older and have comorbidities (i.e., physical or mental disorders) and find it difficult to attend regular follow-ups after the placement of a pessary. Routine disinfection and replacement including close observations for any symptoms or signs of complications are necessary to maintain a pessary. Minor complications include vaginal bleeding, discomfort, vaginal lacerations or erosions, and constipation. Major complications are rare but can be fatal in vulnerable patients. Vesicovaginal fistulas, small bowel obstruction, hydronephrosis, and urosepsis are included as severe complications.

In our case, the neglected pessaries were successfully removed without any complications (i.e., bladder, vagina, or rectal injury) under general anesthesia. A vaginal orifice formed in both patients. The space occupied by the neglected pessary caused granulation as time passed. We suppose that a neglected pessary acts in a similar way to a foreign body migrating in the abdominal cavity before autonomous excretion through the colon. Several cases of retained gauze after abdominal surgery have been described to cause aseptic fibrosis, fusion, and encystment
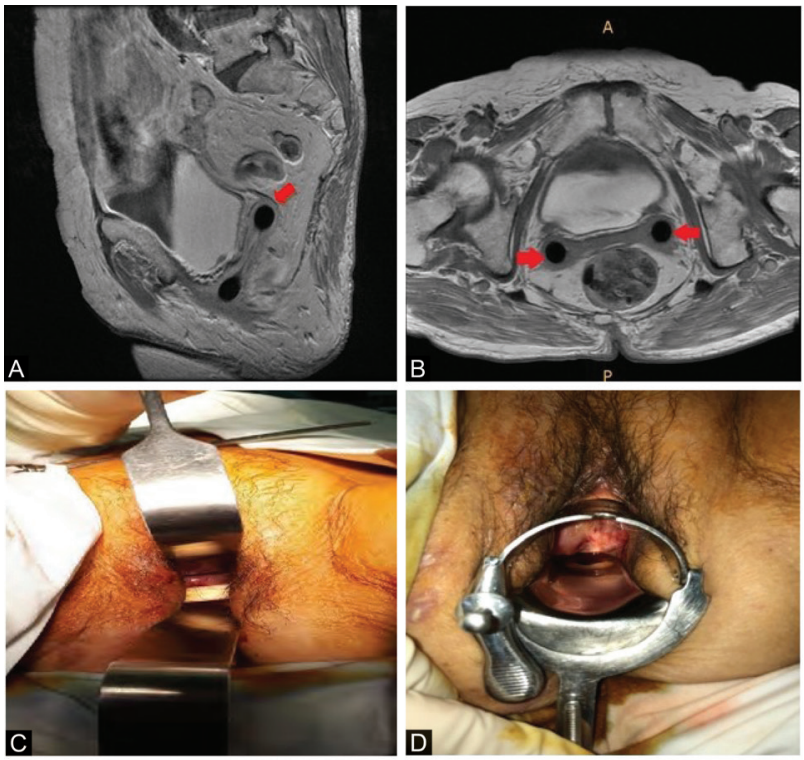

Figure 1. (A) Magnetic Resonance Image (sagittal view), Round latrogenic Material (Pessary) in Vaginal Canal and Rectovaginal space; (B) Magnetic Resonance Image (Axial view), Round latrogenic Material (Pessary) in Rectovaginal Space; (C) Preoperative Image of a Neglected Pessary; (D) Postoperative Image (The removal of pessary).
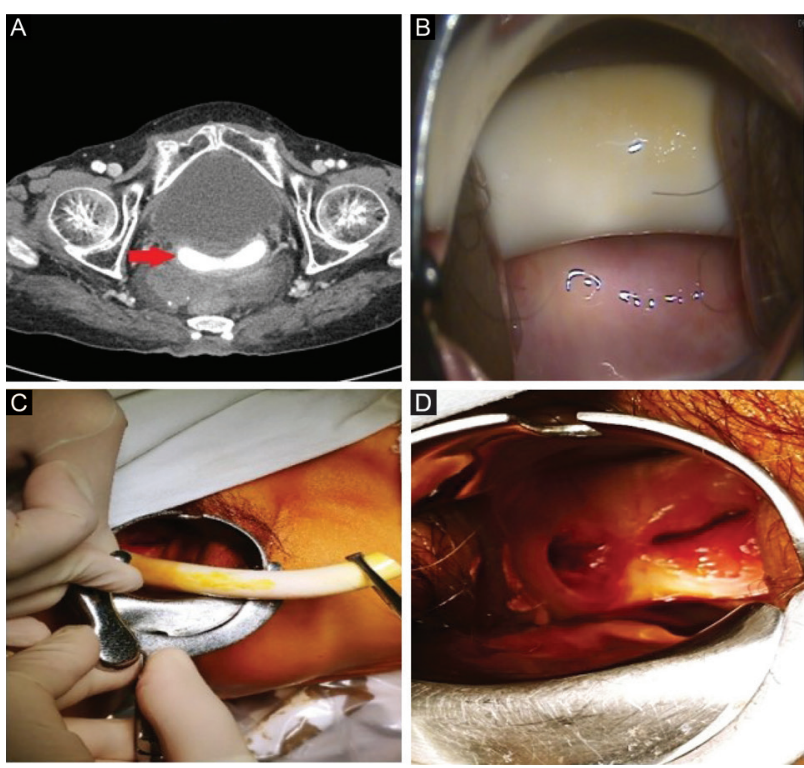

Figure 2. (A) Computerized Tomography Image (Axial view), Round latrogenic Material (Pessary) in Vaginal Canal and Rectovaginal Space; (B) Preoperative Image of a Neglected Pessary; (C) Rotated Removal of a Neglected Pessary; (D) Postoperative Image, (Orifice was observed in the right fornix of vagina).

$(9,10)$. Gauze transmurally migrates into the colon and is excreted by defecation because of those mechanisms.

A vaginal orifice created by a neglected pessary is typically considered a complication, but in our cases, the septa supported the uteropelvic organs. To the best of our knowledge, these are the first cases to report a vaginal orifice as a neglected pessary complication that reduced the protrusion of pelvic structures. We assume this vaginal orifice produced similar outcomes as a 'Le Fort' operation, 
which involves removing parts of the anterior and posterior vaginal wall, followed by sealing the margins of the anterior and posterior walls to each other $(11,12)$. This is probably why the patients did not relapse to a pelvic organ prolapse. The two case patients remained well with no relapse at their last clinic check-ups. Although these are fortunate cases, serious complications can occur due to a neglected pessary. Therefore, physicians must emphasize the importance of routine follow-ups after inserting a pessary.

\section{Authors' Contribution}

HAB wrote the manuscript. JIC and SHC searched related literature and revised manuscript. JHS designed and revised manuscript.

\section{Conflict of Interests}

Authors declare that they have no conflict of interests.

\section{Ethical Issues}

Informed consent was obtained from the patients.

\section{Financial Support}

This study was supported by a research fund from Soonchunhyang University.

\section{References}

1. Thakar R, Stanton S. Management of genital prolapse. BMJ. 2002;324(7348):1258-1262. doi:10.1136/bmj.324.7348.1258

2. Powers K, Lazarou G, Wang A, et al. Pessary use in advanced pelvic organ prolapse. Int Urogynecol J Pelvic Floor Dysfunct.
2006;17(2):160-164. doi:10.1007/s00192-005-1311-8

3. Bash KL. Review of vaginal pessaries. Obstet Gynecol Surv. 2000;55(7):455-460. doi:10.1097/00006254-200007000-00025

4. Alperin $M$, Khan A, Dubina E, et al. Patterns of pessary care and outcomes for medicare beneficiaries with pelvic organ prolapse. Female Pelvic Med Reconstr Surg. 2013;19(3):142-147. doi:10.1097/SPV.0b013e31827e857c

5. Jelovsek JE, Maher C, Barber MD. Pelvic organ prolapse. Lancet. 2007;369(9566):1027-1038. doi:10.1016/s0140-6736(07)604620

6. Bordman R, Telner D. Practice tips. Pessary insertion: choosing appropriate patients. Can Fam Physician. 2007;53(3):424-425.

7. Atnip SD. Pessary use and management for pelvic organ prolapse. Obstet Gynecol Clin North Am. 2009;36(3):541-563. doi:10.1016/j.ogc.2009.08.010

8. Wu V, Farrell SA, Baskett TF, Flowerdew G. A simplified protocol for pessary management. Obstet Gynecol. 1997;90(6):990-994. doi:10.1016/s0029-7844(97)00481-x

9. Choi JW, Lee CH, Kim KA, Park CM, Kim JY. Transmural migration of surgical sponge evacuated by defecation: mimicking an intraperitoneal gossypiboma. Korean J Radiol. 2006;7(3):212-214. doi:10.3348/kjr.2006.7.3.212

10. Modrzejewski A, Kiciak A, Sled冈 M, et al. Migration of a foreign body into the colon and its autonomous excretion. Med Sci Monit. 2011;17(3):CS34-38. doi:10.12659/msm.881438

11. Abbasy S, Kenton K. Obliterative procedures for pelvic organ prolapse. Clin Obstet Gynecol. 2010;53(1):86-98. doi:10.1097/ GRF.0b013e3181cd4252

12. Koski ME, Chow D, Bedestani A, Togami JM, Chesson RR, Winters JC. Colpocleisis for advanced pelvic organ prolapse. Urology. 2012;80(3):542-546. doi:10.1016/j.urology.2012.06.009

(C) 2021 The Author(s); This is an open-access article distributed under the terms of the Creative Commons Attribution License (http:// creativecommons.org/licenses/by/4.0), which permits unrestricted use, distribution, and reproduction in any medium, provided the original work is properly cited. 